\title{
Silencing of argininosuccinate lyase inhibits colorectal cancer formation
}

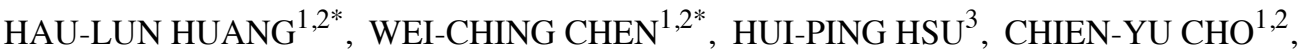 \\ YU-HSUAN HUNG ${ }^{1,2}$, CHIH-YANG WANG ${ }^{1,2}$ and MING-DERG LAI ${ }^{1,2,4}$ \\ ${ }^{1}$ Institute of Basic Medical Sciences, College of Medicine, National Cheng Kung University; \\ Departments of ${ }^{2}$ Biochemistry and Molecular Biology, and ${ }^{3}$ Surgery, College of Medicine, \\ National Cheng Kung University; ${ }^{4}$ Center for Infectious Diseases and Signaling Research, \\ College of Medicine, National Cheng Kung University, Tainan, Taiwan, R.O.C.
}

Received May 19, 2016; Accepted July 14, 2016

DOI: 10.3892/or.2016.5221

\begin{abstract}
Arginine and nitric oxide (NO) are important mediators of tumorigenesis in various types of cancer. Dysregulation of NO content by argininosuccinate lyase (ASL) has been previously demonstrated to inhibit the proliferation of liver and breast cancer cells. However, the function of ASL in colon cancer is not well defined. The present study aimed to determine the effect of ASL on colon cancer. Western blot analysis indicated that ASL expression was induced by endoplasmic reticulum stress in HCT116 and SW480 colon cancer cells. Additionally, the expression of ASL in colon cancer tissues was enhanced compared with that in the adjacent normal tissues, and the patients with colon cancer with higher ASL expression exhibited poorer survival rates. Transfection of ASL-targeting short hairpin RNA (shRNA) into HCT116 cells inhibited cell proliferation and decreased anchorage-independent growth in a soft agar assay. In addition, when injected subcutaneously into NOD/SCID mice, stable transfectant ASL-downregulated HCT116 cells exhibited decreased in vivo tumorigenic ability. Flow cytometric analysis of cell cycle progression indicated that ASL-targeting shRNA induced G2/M arrest, and western blot analysis showed that the inhibition of ASL was accompanied by cyclin A2 degradation. Furthermore, ASL-targeting shRNA resulted in increased autophagosomes and decreased NO levels. Inhibition of NO by the NO synthase inhibitor L-NMMA significantly reduced cell proliferation and colony formation. In summary, the results of the present study indicated that ASL-targeting shRNA-induced growth inhibition
\end{abstract}

Correspondence to: Dr Ming-Derg Lai, Department of Biochemistry and Molecular Biology, College of Medicine, National Cheng Kung University, 1 University Road, Tainan City 701, Taiwan, R.O.C.

E-mail: a1211207@mail.ncku.edu.tw

${ }^{*}$ Contributed equally

Key words: argininosuccinate lyase, nitric oxide, autophagy, endoplasmic reticulum, cyclin A, colorectal cancer is associated with decreased cyclin A2 expression and NO content in colon cancer.

\section{Introduction}

Colorectal cancer is a common disease worldwide and a leading cause of cancer-associated mortality (1). Epidemiological studies have previously indicated that colorectal cancer is associated with metabolic alteration and changes in energy homeostasis $(2,3)$. Patients with a higher body mass index (BMI) have an increased risk of colorectal cancer, and the link between obesity and colorectal carcinogenesis has been demonstrated by meta-analyses (4-6). Furthermore, tumor cells are associated with altered metabolites, as demonstrated in HT29 cells (7). The differential metabolites between colorectal adenoma and non-adenoma tissues have been previously identified by gas chromatography and liquid chromatography time-of-flight mass spectrometry (8), and the alteration of metabolite profiles, including acylcarnitine, glycerophospholipids and arginine, may be used as a biomarker for cancer $(9,10)$.

Arginine, a semi-essential amino acid, mediates multiple cellular functions, including polyamine and nitric oxide (NO) synthesis, in various types of cancer. Arginine supplementation has been shown to increase the risk of colorectal carcinogenesis (11). Deprivation of arginine inhibits DNA synthesis and induces cell apoptosis, and the breakdown of arginine by arginase results in the inhibition of tumor growth (12-14). However, supplement-mediated arginine imbalance causes diverse effects on cancer development. L- and D-arginines were observed to enhance and inhibit tumor growth, respectively, in a transplantation animal tumor model (15). In addition, the concentration of arginine was previously demonstrated to be decreased in the plasma serum of patients with colon, breast and pancreatic cancer (16), whereas patients with colorectal cancer who were provided with L-arginine exhibited reduced crypt cell hyperproliferation, which is a characteristic of colorectal cancer development (17-19). In a previous study, $\mathrm{N}$-hydroxy-L-arginine selectively reduced the proliferation of breast cancer cells with high arginase activity (20). These 
studies indicate that the homeostasis of arginine in cancer requires further investigation.

Arginine is a substrate for NO synthase (NOS), and the production of arginine is catalyzed by the enzymes argininosuccinate synthase (ASS) and argininosuccinate lyase (ASL). ASS catalyzes the conversion of citrulline and aspartate into argininosuccinate, which is then converted to arginine and fumarate by ASL. ASS is highly expressed in ovarian, gastric and colorectal cancer tissues compared with normal tissues. By contrast, the expression of ASS is low in melanoma and hepatocellular carcinoma (21). Downregulation of ASS is associated with chemosensitivity, and the combination of arginine deiminase with chemotherapy enhances the antitumorigenic effect (22). Cancer cells with ASS-deficiency are dependent on extracellular arginine and the drug ADI-PEG has been used against melanoma and hepatocellular carcinoma to deplete the levels of extracellular arginine (23). The pathway of ASS, ASL and NOS contributes to NO production, which mediates signal transduction and acts as an anti-oncogenic target for cancer therapy (24). Decreased NO synthesis has previously been observed in ASS- and ASL-deficient cancer patients with high plasma citrulline (25). ASL is required in the formation of the NO synthesis protein complex, and functions as a key regulator of NO production (26). ASL has been previously reported to promote cancer cell growth via cyclin A2, and NO supplementation rescues cell growth inhibition caused by ASL-targeted short hairpin RNA (shRNA). The induction of ASL by endoplasmic reticulum (ER) stress, caused by the accumulation of misfolded proteins, has been observed in liver and breast cancer $(27,28)$. Activation of intracellular signal transduction by misfolded proteins, termed the unfolded protein response (UPR), affects cell proliferation and is associated with the alteration of amino acid metabolism (29). Targeting ER stress components, including PKR-like ER kinase, inositol-requiring 1 and activating transcription factor 6 , may be a potential therapeutic approach in cancer $(30,31)$.

The present study aimed to investigate the function of ASL in colon cancer and to characterize ASL expression in response to ER stress by exposing colon cancer cells to tunicamycin. The results demonstrated that ASL is overexpressed in colon cancer and that its expression is induced by ER stress. Inhibition of ASL by shRNA decreased colon cancer cell proliferation in vitro and in vivo, and decreased ASL levels were found to be associated with autophagy and NO levels.

\section{Materials and methods}

Reagents, chemicals and antibodies. The following antibodies were utilized in the present study: anti-ASL (H00000435-M01) (Abnova Corporation, Taipei, Taiwan), anti-78 kDa glucose-regulated protein (\#61097) (GRP78; BD Biosciences, Erembodegem, Belgium), anti- $\beta$-actin (MAB1501R) (Chemicon, Pittsburgh, PA, USA), anti-GAPDH (\#610979) (GeneTex, Irvine, CA, USA), anti-cyclin A2 (SC-596), CDK4 (SC-601) and CDK2 (SC-163) (Santa Cruz Biotechnology, Inc., Dallas, TX, USA), anti-cyclin B1 (\#1495-S) and cyclin E1 (\#1655-S) (Epitomics, Burlingame, CA, USA), anti-cyclin D1 (\#2926-S) (Cell Signaling Technology, Inc., Danvers, MA, USA) and anti-microtubule-associated protein 1 light chain $3 \beta$ (LC3B) antibody (L7543) (Sigma-Aldrich, St. Louis, MO,
USA). The ASL plasmid was purchased from OriGene Technologies, Inc. (Rockville, MD, USA).

Cell culture. The human colon cancer cell lines HCT116 and SW480, and the transfectant cells were cultured in Dulbecco's modified Eagle's medium (DMEM) supplemented with $10 \%$ fetal bovine serum, penicillin and streptomycin, at $37^{\circ} \mathrm{C}$ in an atmosphere of $5 \% \mathrm{CO}_{2}$.

Reverse transcription-polymerase chain reaction (RT-PCR) analysis. Total RNA was extracted from cells using TRIzol reagent (MDBio, Inc., Taipei, Taiwan) and the cDNA was synthesized using M-MLV reverse transcriptase (Promega Corporation, Madison, WI, USA). RT-PCR was performed to detect ASL and GAPDH expression using Pro Taq polymerase (Protech Technology Enterprise Co., Ltd., Taipei, Taiwan) on an ABI thermocycler (Applied Biosystems, Thermo Fisher Scientific, Inc., Waltham, MA, USA). The PCR conditions were as follows: initial activation for $5 \mathrm{~min}$ at $94^{\circ} \mathrm{C}$, followed by cycles of denaturation for $30 \mathrm{sec}$ at $94^{\circ} \mathrm{C}$, annealing for $30 \mathrm{sec}$ at $52^{\circ} \mathrm{C}$ (for ASL and GRP78) and extension for $30 \mathrm{sec}$ at $72^{\circ} \mathrm{C}$, and an end step for $7 \mathrm{~min}$ at $72^{\circ} \mathrm{C}$. The primer sequences were as follows: ASL, TGATGCCCCAGAAGAAA AAC and TTTGCGGACCAGGTAATAGG; GAPDH, TGA AGGTCGGTGTGAACGGATTTGGC and CATGTAGGC CATGAGGTCC ACCAC.

Western blot analysis. The protein lysates from cells and clinical cancer tissues were harvested in radioimmunoprecipitation lysis buffer and $35 \mu \mathrm{g}$ protein was loaded into SDS-PAGE gels. Proteins were then transferred to a polyvinylidene difluoride membrane following electrophoretic separation. The membrane was blocked with $5 \%$ milk and probed with specific antibodies. The detection of protein was visualized with a UVP BioSpectrum imaging system (UVP, LLC, Upland, CA, USA).

Tissue samples. The clinical colorectal cancer and adjacent normal tissues were obtained from the National Cheng Kung University Hospital (Tainan, Taiwan) with approval of the Institutional Review Board.

SurvExpress analysis. The overall survival data of the colon cancer patients with high and low ASL expression were obtained from the SurvExpress database (bioinformatica.mty.itesm.mx/SurvExpress). The data were identified from the dataset as 'Colon Metabase Censoring Under Revision' with probe 204608 .

RNA interference (RNAi) by shRNA. The ASL-targeting shRNA was obtained from the National RNAi Core Facility (Academia Sinica, Taipei, Taiwan) and the target sequence was as follows: CACCTTCAAACTGAACTCCAA.

Colony formation assay. The transfectants were plated in 6 -well plates and incubated at $37^{\circ} \mathrm{C}$ in a $5 \% \mathrm{CO}_{2}$ incubator. The number of colonies was counted after 10 days.

Anchorage-independent growth ability. Anchorageindependent growth ability was assessed by soft agar assay. 
A

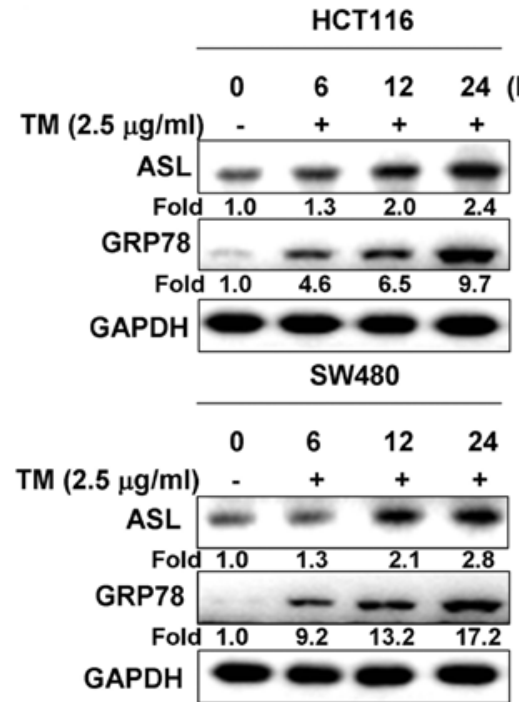

HCT116 ASL protein expression

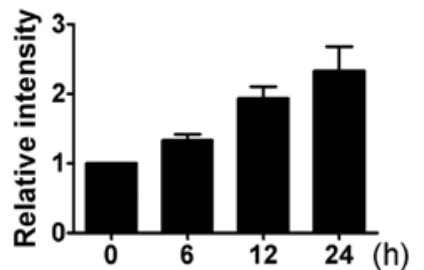

SW480 ASL protein expression

(h)

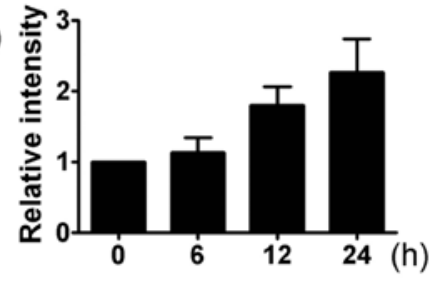

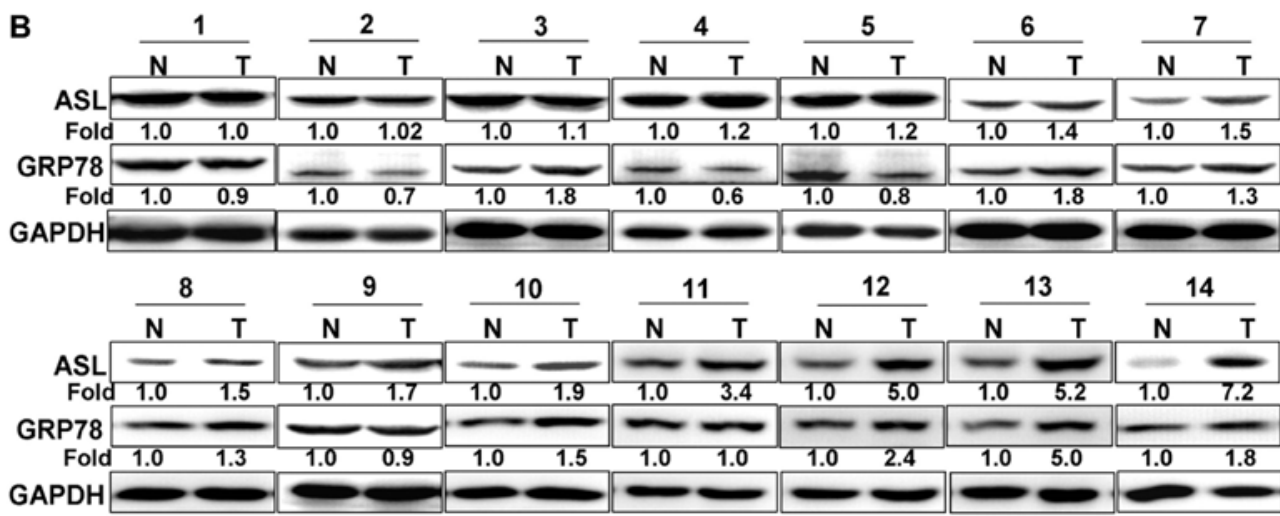

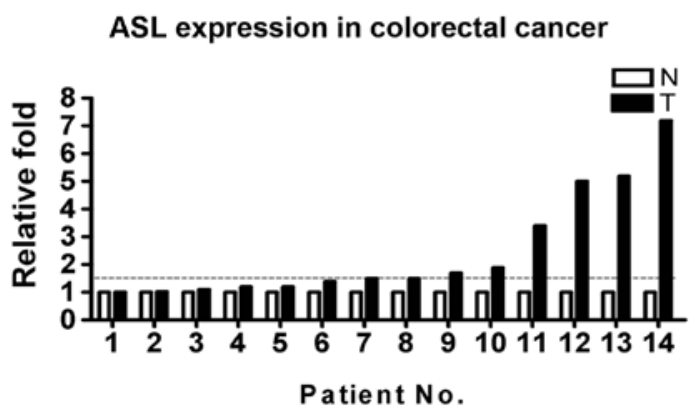

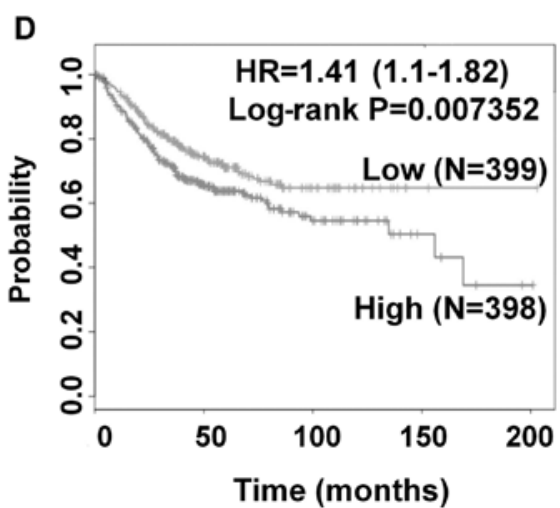

Figure 1. Endoplasmic reticulum stress induces ASL expression in colon cancer. (A) The HCT116 and SW480 colon cancer cell lines were incubated with $2.5 \mu \mathrm{g} / \mathrm{ml}$ tunicamycin for the indicated times. Cell lysates were harvested and the ASL expression level was analyzed by western blotting. The indicated fold-change was quantified by ImageJ software. (B) Protein lysates of colon cancer and adjacent normal tissues were harvested and the expression levels of ASL, GRP78 and GAPDH were determined by western blot analysis. (C) Quantitative fold-changes of ASL are expressed as a bar graph, with the defined threshold of a 1.5-fold-change. (D) Association between ASL expression and survival was identified using the SurvExpress database. ASL, argininosuccinate lyase; GRP78, 78 kDa glucose-regulated protein.

The lower layer contained $0.6 \%$ agar in DMEM, and $5 \times 10^{3}$ cells were mixed with $0.3 \%$ agar and plated in the upper layer. The number of colonies was quantified after incubation for 14 days at $37^{\circ} \mathrm{C}$ in a $5 \% \mathrm{CO}_{2}$ incubator.

Tumorigenicity in NOD/SCID mice. Male NOD/SCID mice (8-weeks old) were obtained from the Animal Center of the National Cheng Kung University. The protocols were approved by the Animal Welfare Committee at National Cheng Kung University. Transfectant cells $\left(2 \times 10^{6}\right)$ were suspended in $0.2 \mathrm{ml}$ DMEM and subcutaneously injected into the NOD/SCID mice. Tumor growth was monitored for 35 days.

Monodansylcadaverine (MDC) staining of autophagy. MDC staining was conducted using a Cayman autophagy/cytotoxicity dual staining kit (Cayman Chemical Co., Ann Arbor, MI, 
A
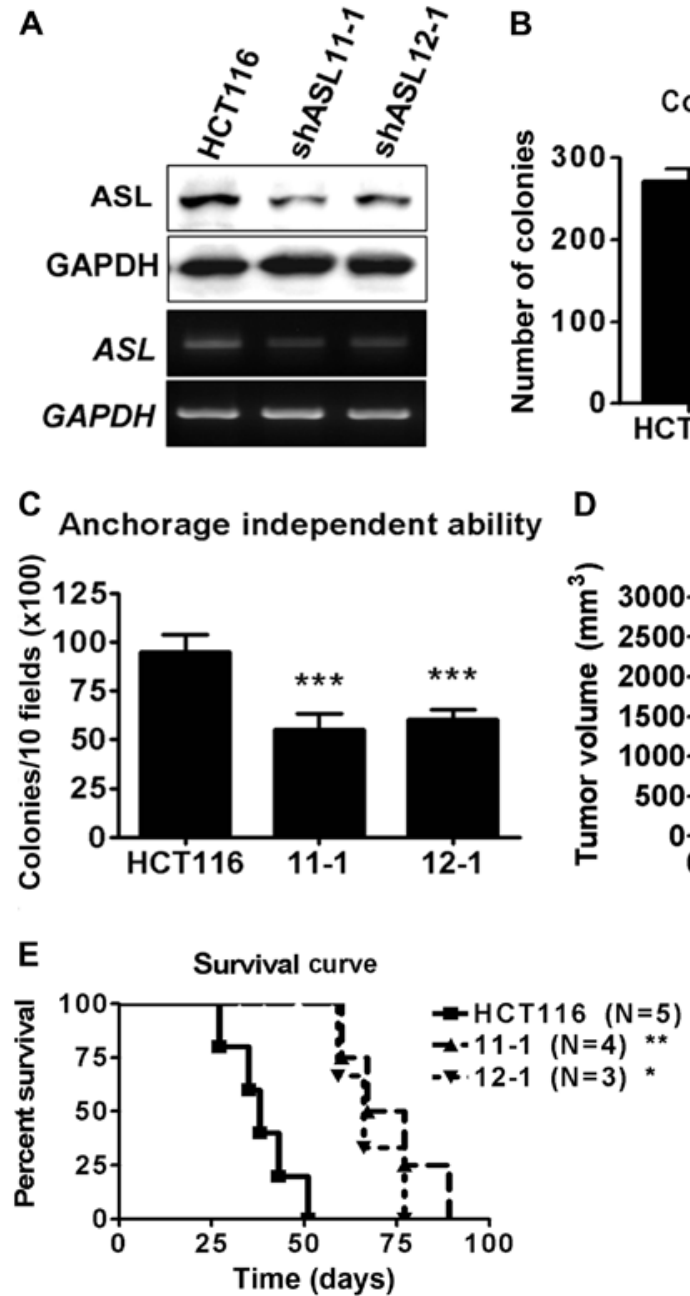

Colony formation assay

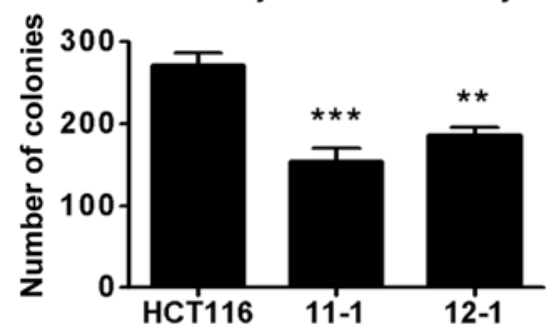

D

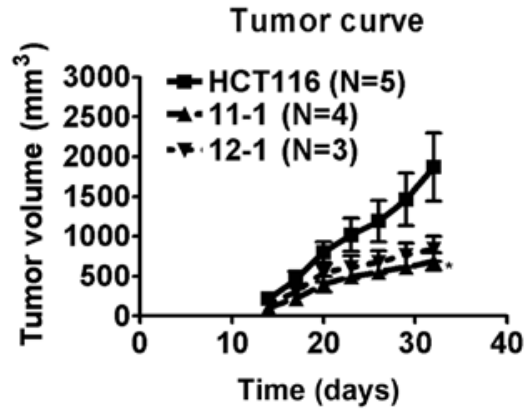

Figure 2. ASL shRNA inhibits colon cancer cell proliferation in vitro and tumor growth in vivo. (A) Expression of ASL in the stable transfectant cells was analyzed by western blot and reverse transcription-polymerase chain reaction analyses. (B) Cell proliferation of the ASL-targeting shRNA transfectants was determined by colony formation assay. (C) Anchorage-independent growth of the transfectants was determined by soft agar assay. (D) Transfectants (2x10 ${ }^{6}$ ) were subcutaneously transplanted into NOD/SCID mice. Tumor volumes were monitored for 35 days. (E) The survival rate of tumor-bearing mice was monitored. Statistical analysis was performed by Student's t-test; ${ }^{*} \mathrm{P}<0.05,{ }^{* *} \mathrm{P}<0.01,{ }^{* * *} \mathrm{P}<0.001$ vs. the parental cell line. ASL, argininosuccinate lyase; shRNA, short hairpin RNA.

USA) according to the manufacturer's instructions. Images were obtained by fluorescence microscopy.

Intracellular arginine content. The concentration of intracellular arginine was analyzed by high performance liquid chromatography (HPLC) analysis using Agilent ZORBAX Eclipse AAA column (cat. no. 993400-902; Agilent Technologies, Inc., Santa Clara, CA, USA).

MTT assay. The transfectants were plated into 24-well plates at the number of $2 \times 10^{4}$. The transfectants were incubated with MTT working solution $(1 \mathrm{mg} / \mathrm{ml})$ at $37^{\circ} \mathrm{C}$ for $4 \mathrm{~h}$ and dimethyl sulfoxide (DMSO) was used to dissolve the crystals. Absorbance was detected at a wavelength of $590 \mathrm{~nm}$ using a spectrophotometer.

Statistical analysis. All results are presented as the mean \pm standard error of the mean. The statistical significance was determined by t-test using Prism software (GraphPad, Inc., La Jolla, CA, USA).

\section{Results}

ASL expression is induced by ER stress and is overexpressed in colorectal cancer tissues. To investigate whether ASL is induced by ER stress, HCT116 and SW480 colorectal cancer cells were treated with tunicamycin. As shown by western blot analysis, tunicamycin significantly increased the ASL expression levels in the HCT116 and SW480 cells (Fig. 1A). To identify the association of ASL with the pathological characteristics of colorectal cancer, ASL protein expression was analyzed in clinical colon cancer and adjacent normal tissues. Western blot analysis indicated that the expression level of ASL was significantly increased in 8 of the 14 paired samples, with the threshold of increased expression defined as a 1.5 -fold change (Fig. $1 \mathrm{~B}$ and $\mathrm{C}$ ). To further elucidate the association of ASL expression with clinical outcome, survival curves from SurvExpress database were analyzed. Colon cancer patients with higher ASL expression exhibited reduced survival rates compared with patients with lower ASL expression (Fig. 1D). 
A

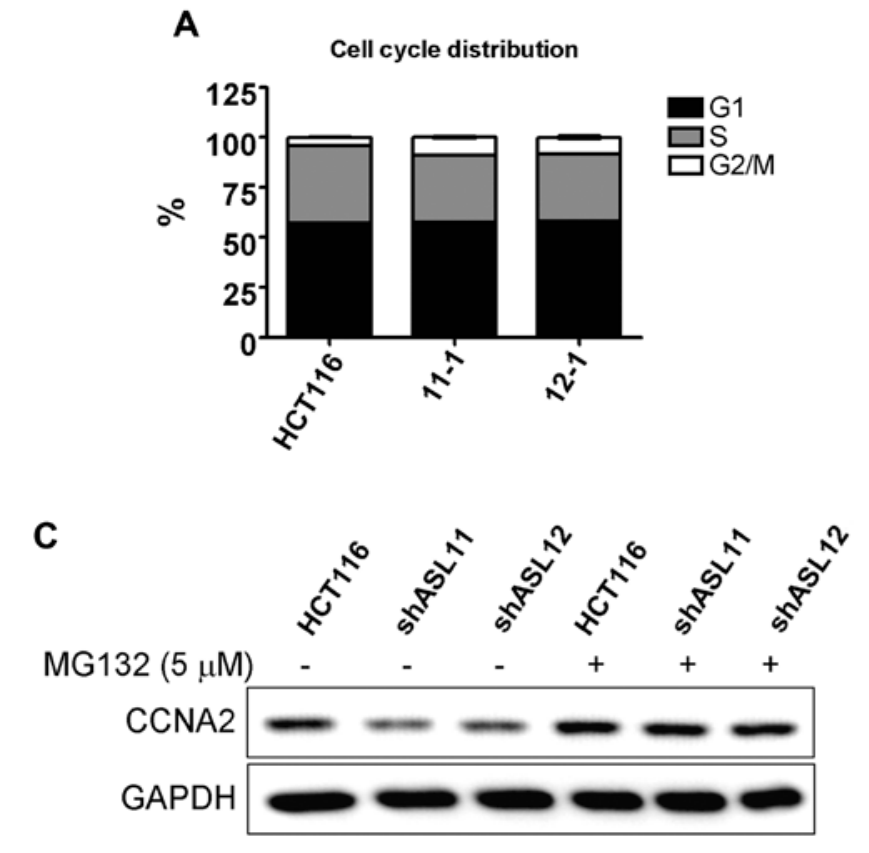

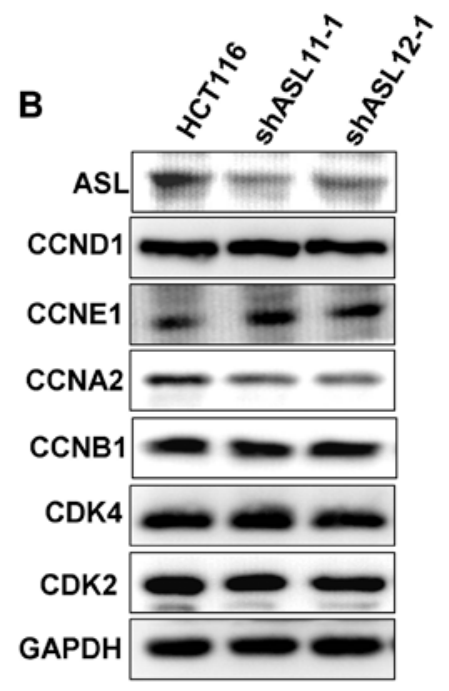

Figure 3. ASL-targeting shRNA induces G2/M phase arrest in colon cancer cells. (A) Cell cycle progression of the ASL-targeting shRNA stable transfectants was analyzed by flow cytometry. (B) Protein lysates of the transfectants were harvested and the levels of cell cycle-associated proteins were analyzed by western blotting. (C) Transfectants were treated with $5 \mu \mathrm{M}$ MG132 and the cyclin A2 was detected by western blotting. ASL, argininosuccinate lyase; shRNA, short hairpin RNA.

ASL shRNA inhibits cell growth in colorectal cancer. To study the function of ASL in colorectal cancer, HCT116 cells were transfected with ASL-targeting shRNA, and the stable transfectant cells were identified by puromycin selection. The expression level of ASL in the stable transfectants was examined by western blot and RT-PCR analyses (Fig. 2A). The stable ASL-targeting shRNA transfectants exhibited decreased anchorage-dependent and anchorage-independent growth ability compared with the parental HCT116 cells (Fig. 2B and C). To determine the effect of ASL on tumorigenic capacity, the transfectants were subcutaneously injected into NOD/SCID mice. ASL-targeting shRNA decreased the rate of tumor growth in vivo (Fig. 2D). In addition, the NOD/SCID mice transplanted with ASL-knockdown HCT116 cells demonstrated prolonged survival (Fig. 2E). These data indicate that RNAi of ASL inhibited the formation of colon cancer.

ASL shRNA decreases cyclin A2 expression in colon cancer. To investigate the mechanism of growth inhibition induced by ASL-targeted shRNA, cell cycle progression was examined. Flow cytometric analysis demonstrated that ASL-targeting shRNA enhanced G2/M phase arrest (Fig. 3A). In addition, western blot analysis demonstrated that HCT116 transfectants with ASL downregulation exhibited decreased cyclin A2 expression levels (Fig. 3B). The decrease in cyclin A2 was partially rescued by MG132 proteasome inhibitor treatment, indicating that decreased cyclin A2 may be a result of alterations to degradation (Fig. 3C).

ASL-targeting shRNA induces autophagy in colon cancer. Autophagy contributes to amino acid maintenance, and ASL-targeting shRNA has been previously demonstrated to induce autophagy in liver and breast cancer cells $(27,28)$. Thus, the present study examined whether ASL-targeting shRNA induced autophagy in colorectal cancer. Western blot analysis indicated that ASL-targeting shRNA promoted the conversion of LC3B-I into LC3B-II (Fig. 4A), and an MDC staining analysis indicated that the HCT116 ASL-targeting shRNA transfectants exhibited enhanced autophagosome synthesis (Fig. 4B). Taken together, these findings demonstrate that downregulation of ASL is associated with autophagy in colorectal cancer.

ASL shRNA decreases NO contents in colon cancer. ASL regulates the regeneration of arginine, which is a substrate required for NO production. Therefore, the present study investigated the effect of ASL on arginine and NO production. The concentration of arginine in the HCT116 shRNA transfectants was analyzed by HPLC analysis. This data indicated that ASL-targeting shRNA did not alter the arginine concentration (Fig. 5A). By contrast, the HCT116 ASL-targeting shRNA transfectants demonstrated decreased NO levels (Fig. 5B). Furthermore, an inhibitor of inducible NOS (iNOS), NG-monomethyl-1-arginine (L-NMMA), significantly reduced NO production, which was similar to the decrease caused by ASL-targeting shRNA (Fig. 5C). In addition, L-NMMA inhibited the growth of cancer cells in cell viability and colony formation assays, suggesting that a reduction in NO mediated by ASL-targeting shRNA may attenuate tumor growth (Fig. 5D and E).

\section{Discussion}

The present study demonstrated that the argininosuccinate lyase (ASL) expression level was enhanced by ER stress in 

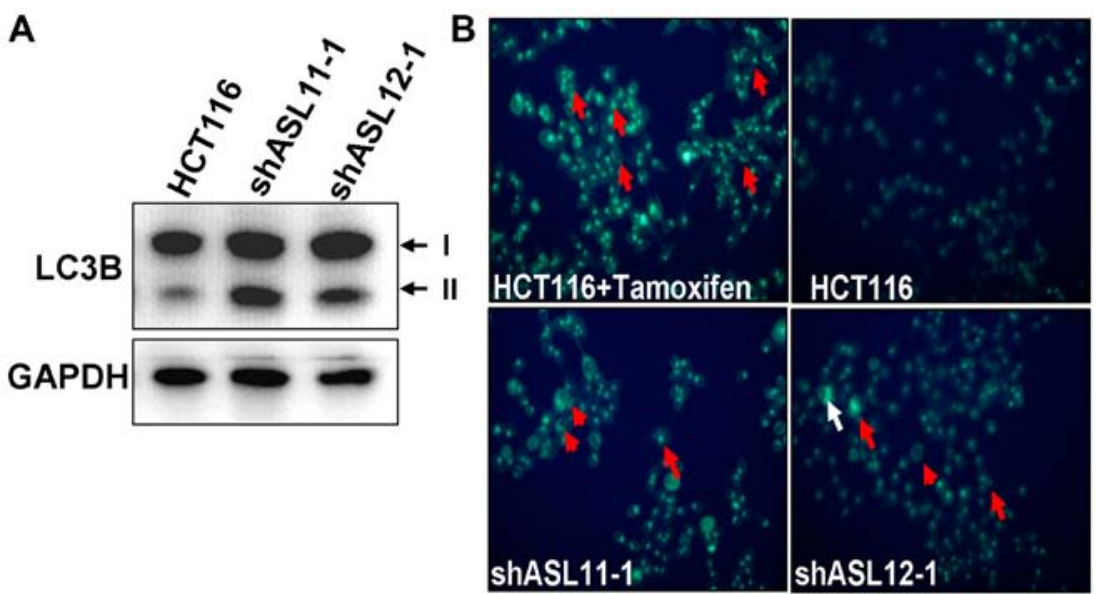

Figure 4. ASL-targeting shRNA induces autophagy in colon cancer. (A) Cell lysates of the stable transfectants were harvested and the LC3B expression levels were analyzed by western blotting. (B) Autophagic vacuoles stained by monodansylcadaverine were detected by fluorescence microscopy (indicated by arrows). ASL, argininosuccinate lyase; shRNA, short hairpin RNA; LC3B, microtubule-associated protein 1 light chain $3 \beta$.

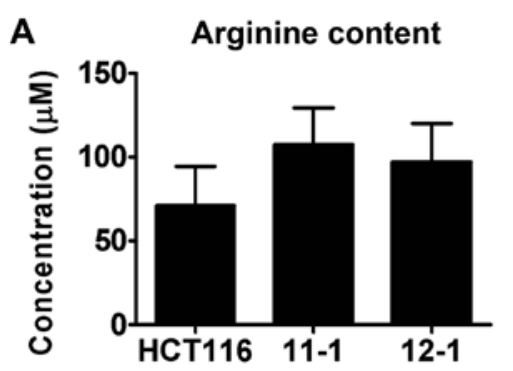

C
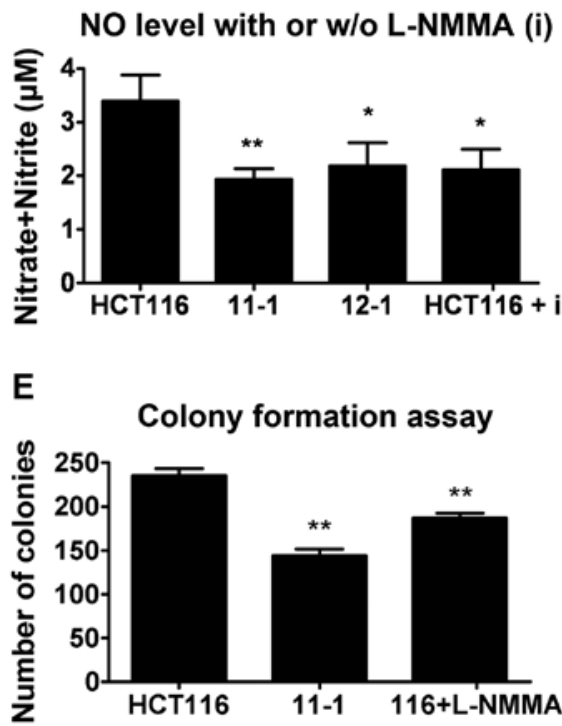

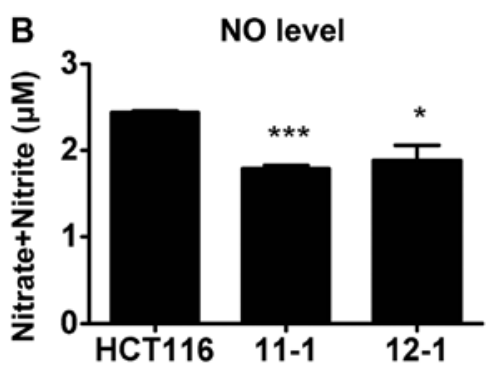

D

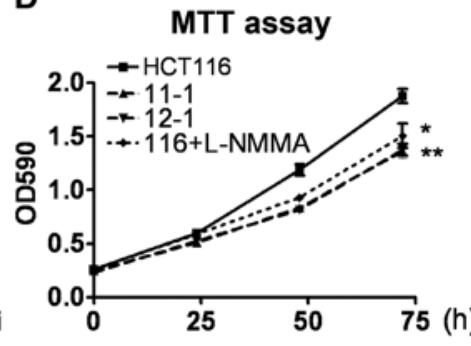

Figure 5. ASL-targeting shRNA decreases NO content in colon cancer. (A) Intracellular arginine in the ASL-targeting shRNA stable transfectant cells was determined by high performance liquid chromatography. (B) NO levels of the cells were analyzed by a nitrate/nitrite colorimetric assay kit. (C) To compare with ASL-targeting shRNA transfectants, parent HCT116 cells were incubated with L-NMMA and the NO level was analyzed by a nitrate/nitrite colorimetric assay kit. (D) Cell proliferation of the transfectant and parental cells with or without L-NMMA treatment was determined by MTT assay. (E) The colonyforming abilities of the transfectant and parental cells were determined in the presence or absence of L-NMMA. Statistical analysis was performed by Student's t-test; ${ }^{*} \mathrm{P}<0.05,{ }^{* *} \mathrm{P}<0.01,{ }^{* * *} \mathrm{P}<0.001$ vs. the parental cells. ASL, argininosuccinate lyase; shRNA, short hairpin RNA; NO, nitric oxide.

colorectal cancer. In addition, the expression of ASL was increased in colorectal cancer tissues compared with the level noted in the adjacent normal tissues, and the prognostic analysis indicated that higher ASL expression was associated with a poor prognosis for survival. ASL-targeting shRNA inhibited cancer cell proliferation in vitro and tumor growth in vivo, and the inhibition of ASL induced cyclin A2 degradation. These data suggest that the effect of ASL on growth 
inhibition may be linked to the degradation of cyclin A2, which results in the $\mathrm{G} 2 / \mathrm{M}$ phase cell cycle arrest. The association between ASL and cyclin A2 in colorectal cancer is in accordance with previous studies that demonstrated the effect of ASL on cyclin A2 in liver and breast cancer $(27,28)$. These findings suggest that ASL may be a potential therapeutic target in colorectal cancer.

Autophagy is a process of degradation of cellular components, and is typically activated in response to intracellular or extracellular stress, including ER stress and starvation (32). The incidence of colon cancer is correlated with the intake of various dietary factors (33), and a previous study demonstrated the dual effect of autophagy on tumor suppression and tumor promotion (34). Autophagy activation is high in colorectal cancer (35), and suppression of autophagy leads to an antitumor effect following activation of UPR signaling (36). By contrast, autophagy has also been previously reported to function as a suppressor of cancer metastasis (37). The present study demonstrated that ASL-targeting shRNA promoted the conversion of LC3B-I to LC3B-II and increased autophagosome formation in colon cancer. Based on the association between ASL and autophagy, targeting arginine metabolic enzymes and autophagy may be a viable anticancer treatment approach.

ASL is involved in arginine production, which is important for protein synthesis and as a substrate for nitric oxide (NO) production. An increased NO level is observed in various types of cancer tissue (38). The induction of NO synthesis by NOS has been previously demonstrated to promote tumor growth, and it has also been shown that NO-generating cancer cells increase neovascularization to facilitate cancer metastasis in vivo (39). However, certain studies have demonstrated an inhibitory effect of NO in cancer. Transfection of a melanoma cell line with iNOS cDNA suppressed tumorigenicity and metastasis $(40,41)$. In addition, the radiation-resistant HT-29 colon cancer cell line was sensitized to radiation by treatment with the NO donor DETANONOate, suggesting that NO donors may have potential as anticancer agents (42). Taken together, these data indicate that NO exerts a dual protumor and antitumor function during cancer progression. The present study demonstrated that ASL-knockdown transfectant cells exhibited decreased NO concentration and reduced tumorigenic ability compared with the parental cells. This suggested that inhibition of NO by ASL-targeting shRNA may contribute to the inhibition of cancer growth. The present study also demonstrated that the cellular arginine level was not altered in the ASL-knockdown transfectant cells. A previous study demonstrated that an increased level of arginine does not alter the NO concentration; however it does restore the NO inhibition induced by glutamine in endothelial cells (43). Arginine administration mediates endothelium-dependent relaxation of blood vessels via NO-dependent or NO-independent pathways, indicating that arginine has multiple functions in physiological regulation (44). The underlying mechanism by which ASL contributes to homeostasis between arginine and NO requires further investigation.

In conclusion, ASL is overexpressed in colorectal cancer and the inhibition of ASL decreases tumor growth. Targeting the metabolic enzyme ASL may be a promising antitumor treatment strategy.

\section{Acknowledgements}

The present study was supported by a grant to M.-D. Lai, (NSC-100-2325-B-006-008) from the National Science Council (Taiwan) and (NHRI-EX100-9927B1) from the National Health Research Institute, Taiwan to establish Centers of Excellence for Cancer Research in Taiwan, (DOH101-TD-C-111-003) Department of Health, Executive Yuan (Taiwan).

\section{References}

1. Shike M, Winawer SJ, Greenwald PH, Bloch A, Hill MJ and Swaroop SV; The WHO Collaborating Centre for the Prevention of Colorectal Cancer: Primary prevention of colorectal cancer. Bull World Health Organ 68: 377-385, 1990.

2. Gerber $\mathbf{M}$ and Corpet D: Energy balance and cancers. Eur $\mathbf{J}$ Cancer Prev 8: 77-89, 1999.

3. Tuominen I, Al-Rabadi L, Stavrakis D, Karagiannides I, Pothoulakis C and Bugni JM: Diet-induced obesity promotes colon tumor development in azoxymethane-treated mice. PLoS One 8: e60939, 2013.

4. Xu X, Zhou L, Miao R, Chen W, Zhou Y, Pang Q, Qu K and Liu C: Association of cancer mortality with postdiagnosis overweight and obesity using body mass index. Oncotarget 7: 5023-5029, 2016

5. Ning Y, Wang L and Giovannucci EL: A quantitative analysis of body mass index and colorectal cancer: Findings from 56 observational studies. Obes Rev 11: 19-30, 2010.

6. Renehan AG, Tyson M, Egger M, Heller RF and Zwahlen M: Body-mass index and incidence of cancer: A systematic review and meta-analysis of prospective observational studies. Lancet 371: 569-578, 2008.

7. Galons JP, Fantini J, Vion-Dury J, Cozzone PJ and Canioni P: Metabolic changes in undifferentiated and differentiated human colon adenocarcinoma cells studied by multinuclear magnetic resonance spectroscopy. Biochimie 71: 949-961, 1989.

8. Nugent JL, McCoy AN, Addamo CJ, Jia W, Sandler RS and Keku TO: Altered tissue metabolites correlate with microbial dysbiosis in colorectal adenomas. J Proteome Res 13: 1921-1929, 2014.

9. Kinross JM, Drymousis P, Jiménez B and Frilling A: Metabonomic profiling: A novel approach in neuroendocrine neoplasias. Surgery 154: 1185-1193, 2013.

10. Giskeødegård GF, Hansen AF, Bertilsson H, Gonzalez SV, Kristiansen KA, Bruheim P, Mjøs SA, Angelsen A, Bathen TF and Tessem MB: Metabolic markers in blood can separate prostate cancer from benign prostatic hyperplasia. Br J Cancer 113: 1712-1719, 2015.

11. Yerushalmi HF, Besselsen DG, Ignatenko NA, BlohmMangone KA, Padilla-Torres JL, Stringer DE, Guillen JM, Holubec H, Payne CM and Gerner EW: Role of polyamines in arginine-dependent colon carcinogenesis in $\mathrm{Apc}^{\mathrm{Min} / \mathrm{+}}$ mice. Mol Carcinog 45: 764-773, 2006.

12. Lamb J and Wheatley DN: Single amino acid (arginine) deprivation induces G1 arrest associated with inhibition of cdk4 expression in cultured human diploid fibroblasts. Exp Cell Res 255: 238-249, 2000.

13. Bach SJ and Swaine D: The Effect of arginase on the retardation of tumour growth. Br J Cancer 19: 379-386, 1965.

14. Yeatman TJ, Risley GL and Brunson ME: Depletion of dietary arginine inhibits growth of metastatic tumor. Arch Surg 126: 1376-1382, 1991.

15. Szende B, Tyihák E and Trézl L: Role of arginine and its methylated derivatives in cancer biology and treatment. Cancer Cell Int 1: 3, 2001

16. Vissers YL, Dejong CH, Luiking YC, Fearon KC, von Meyenfeldt MF and Deutz NE: Plasma arginine concentrations are reduced in cancer patients: Evidence for arginine deficiency? Am J Clin Nutr 81: 1142-1146, 2005.

17. Ma Q, Williamson KE, O'rourke D and Rowlands BJ: The effects of l-arginine on crypt cell hyperproliferation in colorectal cancer. J Surg Res 81: 181-188, 1999.

18. Ma Q, Wang Y, Gao X, Ma Z and Song Z: L-arginine reduces cell proliferation and ornithine decarboxylase activity in patients with colorectal adenoma and adenocarcinoma. Clin Cancer Res 13: 7407-7412, 2007. 
19. Motoo Y, Taga K, Su SB, Xie MJ and Sawabu N: Arginine induces apoptosis and gene expression of pancreatitis-associated protein (PAP) in rat pancreatic acinar AR4-2J cells. Pancreas 20: 61-66, 2000.

20. Singh R, Pervin S, Karimi A, Cederbaum S and Chaudhuri G: Arginase activity in human breast cancer cell lines: N(omega)hydroxy-L-arginine selectively inhibits cell proliferation and induces apoptosis in MDA-MB-468 cells. Cancer Res 60 3305-3312, 2000.

21. Delage B, Fennell DA, Nicholson L, McNeish I, Lemoine NR, Crook T and Szlosarek PW: Arginine deprivation and argininosuccinate synthetase expression in the treatment of cancer. Int $\mathrm{J}$ Cancer 126: 2762-2772, 2010.

22. Liu J, Ma J, Wu Z, Li W, Zhang D, Han L, Wang F, Reindl KM, $\mathrm{Wu} \mathrm{E}$ and $\mathrm{Ma} \mathrm{Q}$ : Arginine deiminase augments the chemosensitivity of argininosuccinate synthetase-deficient pancreatic cancer cells to gemcitabine via inhibition of NF- $\kappa \mathrm{B}$ signaling. BMC Cancer 14: 686, 2014.

23. Ensor CM, Holtsberg FW, Bomalaski JS and Clark MA: Pegylated arginine deiminase (ADI-SS PEG $_{20,000 \mathrm{mw}}$ ) inhibits human melanomas and hepatocellular carcinomas in vitro and in vivo. Cancer Res 62: 5443-5450, 2002.

24. Huerta S, Chilka S and Bonavida B: Nitric oxide donors: Novel cancer therapeutics (Review). Int J Oncol 33: 909-927, 2008.

25. Nagasaka $H$, Tsukahara $H$, Yorifuji T, Miida T, Murayama K, Tsuruoka T, Takatani T, Kanazawa M, Kobayashi K, Okano Y, et al: Evaluation of endogenous nitric oxide synthesis in congenital urea cycle enzyme defects. Metabolism 58: 278-282, 2009.

26. Erez A, Nagamani SC, Shchelochkov OA, Premkumar MH, Campeau PM, Chen Y, Garg HK, Li L, Mian A, Bertin TK, et al: Requirement of argininosuccinate lyase for systemic nitric oxide production. Nat Med 17: 1619-1626, 2011.

27. Huang HL, Hsu HP, Shieh SC, Chang YS, Chen WC, Cho CY, Teng CF, Su IJ, Hung WC and Lai MD: Attenuation of argininosuccinate lyase inhibits cancer growth via cyclin A2 and nitric oxide. Mol Cancer Ther 12: 2505-2516, 2013.

28. Huang HL, Chen WC, Hsu HP, Cho CY, Hung YH, Wang CY and Lai MD: Argininosuccinate lyase is a potential therapeutic target in breast cancer. Oncol Rep 34: 3131-3139, 2015.

29. Harding HP, Zhang Y, Zeng H, Novoa I, Lu PD, Calfon M, Sadri N, Yun C, Popko B, Paules R, et al: An integrated stress response regulates amino acid metabolism and resistance to oxidative stress. Mol Cell 11: 619-633, 2003.

30. Luo B and Lee AS: The critical roles of endoplasmic reticulum chaperones and unfolded protein response in tumorigenesis and anticancer therapies. Oncogene 32: 805-818, 2013.

31. Wang Y, Alam GN, Ning Y, Visioli F, Dong Z, Nör JE and Polverini PJ: The unfolded protein response induces the angiogenic switch in human tumor cells through the PERK/ATF4 pathway. Cancer Res 72: 5396-5406, 2012.
32. He C and Klionsky DJ: Regulation mechanisms and signaling pathways of autophagy. Annu Rev Genet 43: 67-93, 2009.

33. Willett WC: Diet and cancer: An evolving picture. JAMA 293: 233-234, 2005.

34. Burada F, Nicoli ER, Ciurea ME, Uscatu DC, Ioana M and Gheonea DI: Autophagy in colorectal cancer: An important switch from physiology to pathology. World J Gastrointest Oncol 7: 271-284, 2015

35. Sato K, Tsuchihara K, Fujii S, Sugiyama M, Goya T, Atomi Y, Ueno T, Ochiai A and Esumi H: Autophagy is activated in colorectal cancer cells and contributes to the tolerance to nutrient deprivation. Cancer Res 67: 9677-9684, 2007.

36. Sakitani K, Hirata Y, Hikiba Y, Hayakawa Y, Ihara S, Suzuki H, Suzuki N, Serizawa T, Kinoshita H, Sakamoto K, et al: Inhibition of autophagy exerts anti-colon cancer effects via apoptosis induced by p53 activation and ER stress. BMC Cancer 15: 795 , 2015.

37. Gozuacik D and Kimchi A: Autophagy as a cell death and tumor suppressor mechanism. Oncogene 23: 2891-2906, 2004.

38. Forbes TA, Hopkins L, Schneider B, Lazarus L, Leitenberg D, Constant S, Schwartz A, Patierno S and Ceryak S: Potential role of nitric oxide in chromium-induced lung carcinogenesis. Cancer Res 11 (Suppl 8): 5456, 2012.

39. Jenkins DC, Charles IG, Thomsen LL, Moss DW, Holmes LS, Baylis SA, Rhodes P, Westmore K, Emson PC and Moncada S: Roles of nitric oxide in tumor growth. Proc Natl Acad Sci USA 92: 4392-4396, 1995.

40. Xie K, Huang S, Dong Z, Juang SH, Gutman M, Xie QW, Nathan $\mathrm{C}$ and Fidler IJ: Transfection with the inducible nitric oxide synthase gene suppresses tumorigenicity and abrogates metastasis by K-1735 murine melanoma cells. J Exp Med 181: 1333-1343, 1995.

41. Juang SH, Xie K, Xu L, Shi Q, Wang Y, Yoneda J and Fidler IJ: Suppression of tumorigenicity and metastasis of human renal carcinoma cells by infection with retroviral vectors harboring the murine inducible nitric oxide synthase gene. Hum Gene Ther 9: 845-854, 1998.

42. Hibbs JB Jr, Taintor RR and Vavrin Z: Macrophage cytotoxicity: Role for L-arginine deiminase and imino nitrogen oxidation to nitrite. Science 235: 473-476, 1987.

43. Arnal JF, Münzel T, Venema RC, James NL, Bai CL, Mitch WE and Harrison DG: Interactions between L-arginine and L-glutamine change endothelial NO production. An effect independent of NO synthase substrate availability. J Clin Invest 95: 2565-2572, 1995.

44. Pieper GM: Review of alterations in endothelial nitric oxide production in diabetes: Protective role of arginine on endothelial dysfunction. Hypertension 31: 1047-1060, 1998. 